\title{
Bilateral Preiser's disease in a patient with hypercoagulability
}

John McMurtry, Jessica Frankenhoff*

Department of Orthopedic Surgery, VCU Medical Center, Richmond, VA, United States

Received: February 22, 2019

DOI: $10.5430 /$ css.v5n $1 \mathrm{p} 20$
Accepted: April 1, 2019

URL: https://doi.org/10.5430/css.v5n1p20

\begin{abstract}
Reports of bilateral Preiser's disease-avascular necrosis of the scaphoid - are rare with only 4 cases reported in the English literature. We present the fifth reported case of bilateral Preiser's disease in the setting of a previously undiagnosed hypercoagulability disorder.
\end{abstract}

Key Words: Preiser's disease, Hypercoagulable, AVN scaphoid

\section{INTRODUCTION}

Avascular necrosis (AVN), is defined as death of bone caused by disruption of the blood supply. AVN of the scaphoid, absent a primary fracture, is called Preiser's disease, after Georg Preiser. ${ }^{[1]}$ As a retrospective review of his original 1910 article points out, in all 5 of his cases there was a fracture of the scaphoid. ${ }^{[2]}$ Despite this, the term Preiser's Disease has become synonymous with AVN of the scaphoid without previous fracture.

The etiology of osteonecrosis avascular necrosis of the scaphoid is unknown. Hypotheses for the disruption of blood flow include "extrinsic factors" (repetitive trauma, steroid use, chemotherapy, alcohol intake) and "intrinsic factors" (genetic, biochemical and systemic: e.g., hypercholesterolemia, connective tissue disorders). ${ }^{[3]}$ A combination of these factors is likely at play, akin to the "two-hit" phenomenon associated with cancer occurrence.

Bilateral Preiser's disease is rare, with only 4 case reports known in the English literature. ${ }^{[3-6]}$ Due to its rarity, the epidemiology of bilateral AVN of the scaphoid is not known. We postulate that an underlying metabolic disorder may be associated with bilateral AVN of the scaphoid. We report on a case of bilateral Preiser's disease in the setting of Factor 5 Leiden heterozygosity and low-Activated Protein C (APC). To our knowledge, there has never been a reported association of hypercoagulability with AVN of the scaphoid, which makes this case unique.

\section{Case report}

A 50-year-old right-hand dominant female presented with an 8-month history of left-sided wrist pain after a minor fall. She had worn a splint since the injury and was living with the pain due to lack of insurance. Her medical history was significant for COPD for which she was given steroid tapers a few times a year for several years. She also suffered from depression. Her medications at admission included ibuprofen, Advair and Ventolin inhalers, Effexor, Clarinex, vitamins and "nutritional supplements". She had no drug allergies and was a 40-year $1 / 2$ ppd smoker, quitting a year

\footnotetext{
${ }^{*}$ Correspondence: Jessica Frankenhoff; Email: jessica.frankenhoff@vcuhealth.org; Address: Department of Orthopedic Surgery, VCU Medical Center, Richmond, VA, United States.
} 
before presentation. She was an unemployed truck driver.

Examination was difficult due to exaggerated pain response with guarding. She had limited range of motion in flexion and extension both actively and passively to about 30 degrees, respectively. Tenderness was significantly worse radially. XiScan images of the scaphoid demonstrated diffuse sclerosis consistent with AVN.

She was sent for formal plain films, which demonstrated fragmentation and irregularity of the proximal pole (see Figure 1). An MRI of the left wrist was performed in December of 2012, showing micro-fractures in both the distal pole and the waist with definite AVN of the proximal pole and mild AVN of the distal pole. She also had moderate radio-carpal degeneration consistent with stage-4 disease from Herbert and Lanzetta's 4-stage radiographic classification ${ }^{[7]}$ (see Table 1). The patient declined steroid injections and underwent an uncomplicated proximal row carpectomy in March 2013. She did well post-operatively until July 2013, when she fell up a stair, hitting her right, previously asymptomatic wrist. She had definite radial-sided swelling on exam, no bruising but diffuse tenderness over the radial side of her wrist. Range of motion was again limited by pain and guarding. Xi-Scan imaging demonstrated a diffusely sclerotic scaphoid and an MRI was obtained. The MRI from November 2013 confirmed a diagnosis of Preiser's with “extensive abnormal signal within the scaphoid with the replacement of the majority of fat signal by fluid signal ... consistent with AVN" (see Figure 2). Based on the MRI imaging studies of both wrists, she had Kalainov's Type I classification of Preiser's disease with necrosis or ischemia of $100 \%$ of the scaphoid with its inherent propensity for scaphoid deterioration. ${ }^{[8]}$ Kalainov's classification dichotomizes Preiser's into Type I with $100 \%$ of the scaphoid avascular, and Type II with less than $50 \%$ of the bone with avascularity.

Table 1. Classifications of avascular necrosis of the scaphoid as published by Herbert and Lanzetta ${ }^{[7]}$

\begin{tabular}{ll}
\hline \multicolumn{1}{l}{ Herbert and Lanzetta Classification } \\
\hline Stage I & $\begin{array}{l}\text { Normal plain films with abnormal } \\
\text { bone scan or MRI } \\
\text { Proximal pole increased density } \\
\text { changes with osteopenia }\end{array}$ \\
Stage II & $\begin{array}{l}\text { Proximal pole fragmentation with or } \\
\text { without pathologic fracture }\end{array}$ \\
Stage III & Carpal collapse and arthritis \\
\hline
\end{tabular}

Since it is unusual to present with bilateral Preiser's disease, even in the setting of a smoker with a history of steroid use, another cause for her bilateral AVN was sought with a hypercoagulability work-up. Interestingly, this found that she was

Published by Sciedu Press
Factor V Leiden heterozygous with a low-Activated Protein C. According to the hematology-oncology, this put her at a tenfold increased risk of venous clotting compared to the average person.

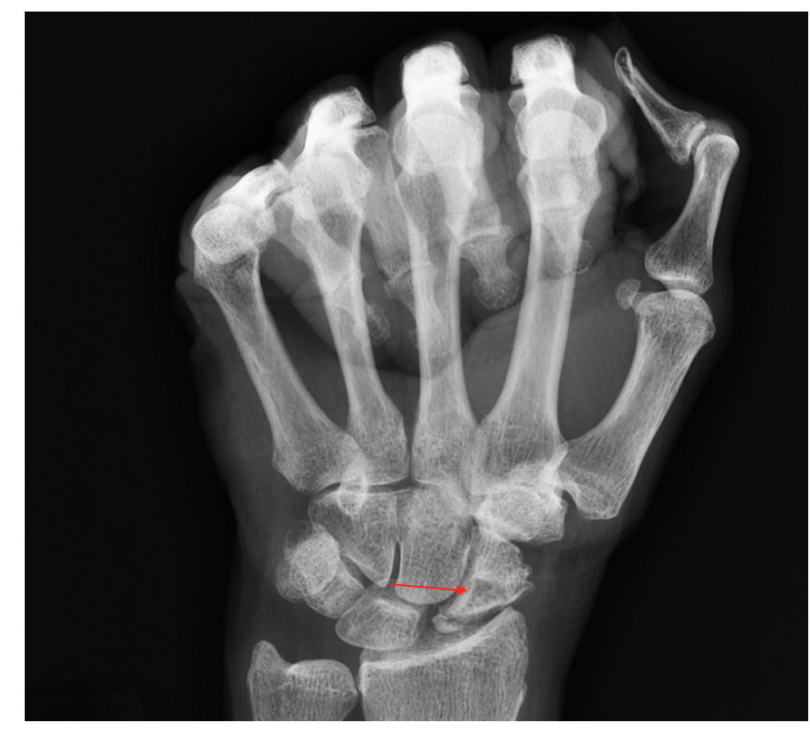

Figure 1. Posteroanterior plain film of the left wrist demonstrating significant irregularity and fragmentation of the scaphoid (arrow)

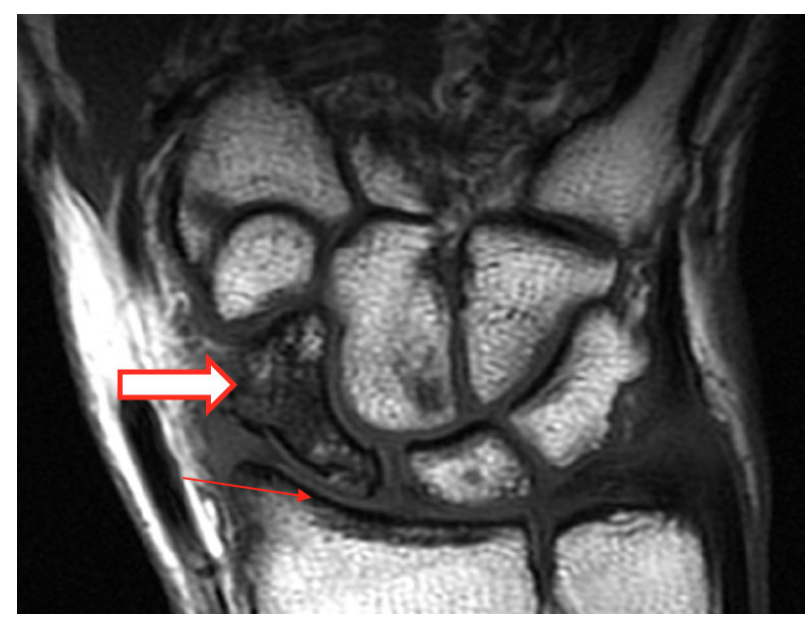

Figure 2. Posteroanterior MRI of the right wrist with AVN of the right scaphoid (large arrow) and early radio-carpal arthritis (small arrow)

She went on to have a steroid shot in her right radio-carpal joint and is waiting for her social situation to improve before contemplating surgical intervention for her right wrist.

\section{Discussion}

Upon initial evaluation, the exact mechanism underlying AVN must be differentiated into traumatic versus atraumatic etiologies. The suspected causes of atraumatic AVN are 
diverse and include intraosseous and extraosseous congenital vascular abnormalities, vasculitis of tenuous blood flow, thrombosis, and fat embolus. In the setting of these abnormalities, additional risk factors such as smoking tobacco, alcoholism, corticosteroid use, myelodysplastic syndromes, vasculitis, hypercholesterolemia, hemoglobinopathies, female gender, and SLE put patients at an increased risk of AVN. ${ }^{[6,9,10]}$ For overall risk of atraumatic AVN, the use of steroids demonstrates the greatest correlation, but incidence of avascular necrosis when steroids are an isolated risk factor is exceedingly rare. ${ }^{[9]}$ This contrasts with situations of systemic illness related to immune complex deposition and vasculitides where steroid use dramatically increases the incidence of avascular necrosis. ${ }^{[9]}$ The role of steroids causing avascular necrosis is difficult to elucidate due to the common comorbidities for which steroids are administered, but steroid use has demonstrated a cumulative negative effect with regards to osseous blood supply. ${ }^{[11]}$ Considering this, patients with both long-term steroid use and use of long-acting steroids associated with certain co-morbidities demonstrate increased observations of avascular necrosis. ${ }^{[9]}$

Tobacco smoking is another demonstrated risk factor with as little as one cigarette per day increasing the risk of AVN. ${ }^{[12]}$ There is no identified significant cumulative effect from tobacco smoking, but it is an independent risk factor imparting a relative risk of 3.0-3.9. ${ }^{[12,13]}$ Alcohol abuse was also found to be a risk factor for avascular necrosis, although it was found to demonstrate a dose-dependent relationship. ${ }^{[14]} \mathrm{Pa}-$ tients with a diagnosis of avascular necrosis demonstrated significantly elevated serum and urinary free cortisol levels, which is thought to contribute to the inherent pathology. ${ }^{[15]}$ Hyperlipidemia was also associated with osteonecrosis, as patients with AVN demonstrated significantly elevated levels of serum cholesterol compared to age-matched controls. ${ }^{[16]}$

Coagulation abnormalities have been implicated in the pathogenesis of avascular necrosis and have come to the forefront of discussion. The pathophysiology related to coagulation abnormalities has several explanations, with no consensus. It is postulated that intravascular coagulation begins in the vul- nerable subchondral microcirculation, which in turn causes vasoconstriction and impaired secondary fibrinolysis. ${ }^{[17]}$ It has also been suggested that thrombosis of venous outflow causes increased intramedullary pressure, which in turn leads to reduced osseous perfusion, hypoxia/anoxia and infarction. ${ }^{[18]}$ Pathology of osteonecrosis demonstrates increased intraosseous pressure as well as evidence of fibrin thrombi within the transitional zone. ${ }^{[17]}$ Several investigations have noted that a large percentage of patients with AVN demonstrate at least one coagulation disorder, but this is often in addition to another systemic risk factor. ${ }^{[17,19]}$

Many different coagulopathies have been tied to avascular necrosis, of which the Factor V Leiden mutation was identified as a potential risk factor. ${ }^{[20]}$ Factor $\mathrm{V}$ Leiden mutation results in resistance to activated protein $\mathrm{C}$, which normally cleaves Factor V. Factor V normally binds to factor Xa leading to the activation of thrombin. There are several other conditions that alter protein $\mathrm{C}$ activity, but Factor $\mathrm{V}$ Leiden is unique in its resistance to activated protein C. ${ }^{[21]}$ Additionally, hypercoagulability has been documented with steroid use and is thought to place the patient in a "prethrombotic state". ${ }^{[22]}$ These findings suggest that patients with a coagulation disorder are at an increased risk of avascular necrosis with concomitant steroid use. ${ }^{[17]}$ Coagulopathies are thought to play a key role in the pathogenesis of osteonecrosis, and it is recommended that all patients with idiopathic etiologies of osteonecrosis and prior corticosteroid use undergo evaluations of coagulation markers. ${ }^{[20]}$

When evaluating our patient, her numerous risk factors, including chronic steroid use, smoking, and coagulopathy all must be considered together as leading to one pathology. Given the propensity for increased coagulation with steroid use combined with her baseline coagulopathy, this appears to be the primary cause of her bilateral scaphoid avascular necrosis. A hypercoagulable work up should be kept in mind in the setting of Preiser's disease.

\section{CONFlicts of InTEREST Disclosure}

The authors declare they have no conflicts of interest.

\section{REFERENCES}

[1] Preiser G. Eine typishe posttraumatische and zur spontanfraktur furende Osteitis des Naviculare carpi. Fortschr Geb Rontgenstr. 1910; 15: 189-97

[2] Kallen AM, Strackee SD. On the History and definition of Preiser t Disease. J Hand Surg(E). 2013; 0E(0): 1-7.

[3] Amillo-Garayoa S, Romero-Munoz LM, Pons-DeVillanueva J. Bilateral Preiserla Preiser's Disease: a case report and review of the literature. Musculoskelet Surg. 2001; 95: 131-133. PMid:21409500. https://doi.org/10.1007/s12306-011-0095-x

[4] Guelpa G, Chamay A, Lagier R. Bialteral Osteochondritis dissecans of the carpal scaphoid. Int Orthop. 1980; 4: 25-30. PMid:7399777.

[5] Imam S, Aldridge C, Lyall H. Bilateral Idiopathic avascular necrosis of the scaphoid. A rare case of Preiserla Disease. J Bone and Joint Surg. 2009; 91: 1400-2.

[6] Lenoir H, Coulet B, Lazerges C, et al. Idiopathic avascular necro- 
sis of the scaphoid: 10 new cases and a review of the literature. Indication for Periserio disease. Orthopedics and Traumatology: Surgery and Research. 2012; 98: 390-387. PMid:22608001. https://doi.org/10.1016/j.otsr.2011.11.013

[7] Herbert TJ, Lanzetta M. Idiopathic avascular necrosis of the scaphoid. J Hand Surg Br. 1994; (2): 174-182.

[8] Kalainov DM, Cohen MS, Hendrix RW, et al. Preiser's disease:identification of two patterns. J. Hand Surg. 2003; 28(5): 767768. https : //doi .org/10.1016/S0363-5023(03)00260-0

[9] Assouline-Dayan Y, Chang C, Greenspan A, et al. Pathogenesis and natural history of osteonecrosis. In Seminars in arthritis and rheumatism. WB Saunders. 2002; 32(2): 94-124.

[10] Urman JD, Abeles M, Houghton AN, et al. Aseptic necrosis presenting as wrist pain in SLE. Arthritis \& Rheumatism. 1977; 20(3): 825-828. https ://doi .org/10.1002/art.1780200311

[11] Fink JC, Leisenring WM, Sullivan KM, et al. Avascular necrosis following bone marrow transplantation: a case-control study. Bone. 1998; 22(1): 67-71. https : //doi.org/10.1016/S8756-3 282 (97) 00219-6

[12] Matsuo K, Hirohata T, Sugioka Y, et al. Influence of alcohol intake, cigarette smoking, and occupational status on idiopathic osteonecrosis of the femoral head. Clinical Orthopaedics and Related Research. 1988; 234: 115-123.

[13] Chang CC, Greenspan A, Gershwin ME. Osteonecrosis: current perspectives on pathogenesis and treatment. In Seminars in arthritis and rheumatism. WB Saunders. 1993; 23(1): 47-69.

[14] Mehsen N, Barnetche T, Redonnet-Vernhet I, et al. Coagulopathies frequency in aseptic osteonecrosis patients. Joint Bone Spine. 2009; 76(2): 166-169. PMid:19211287. https ://doi.org/10.1016/j . jbspin. 2008.04.019
[15] Rico H, Gomez-Castresana F, Cabranes JA, et al. Increased blood cortisol in alcoholic patients with aseptic necrosis of the femoral head. Calcified Tissue International. 1985; 37(6): 585-587. https : //doi.org/10.1007/BF02554910

[16] Moskal JT, Topping RE, Franklin LL. Hypercholesterolemia: an association with osteonecrosis of the femoral head. American Journal of Orthopedics (Belle Mead, NJ). 1997; 26(9): 609-612.

[17] Jones LC, Mont MA, Le TB, et al. Procoagulants and osteonecrosis. The Journal of Rheumatology. 2003; 30(4): 783-791.

[18] Glueck CJ, Freiberg RA, Fontaine RN, et al. Hypofibrinolysis, thrombophilia, osteonecrosis. Clinical Orthopaedics and Related Research. 2001; 386: 19-33. https://doi.org/10.1097/00003086-200 105000-00004

[19] Glueck CJ, Freiberg R, Tracey T, et al. Thrombophilia and hypofibrinolysis. Pathophysiologies of osteonecrosis. Clin Orthop. 1997; 334 43-56. PMid:9005895.

[20] Orth P, Anagnostakos K. Coagulation abnormalities in osteonecrosis and bone marrow edema syndrome. Orthopedics. 2013; 36(4): 290300. PMid:23590772. https : //doi .org/10.3928/01477447-2 0130327-08

[21] Nicolaes GA, Dahlb AB. Activated protein C resistance and thrombosis: factor $\mathrm{V}$ mutations causing hypercoagulable states. Hematology/Oncology Clinics of North America. 2003; 17(1): 37-61. https : //doi.org/10.1016/S0889-8588(02)00097-7

[22] Cosgriff SW. Thromboembolic complications associated with ACTH and cortisone therapy. Journal of the American Medical Association. 1951; 147(10): 924-926. https ://doi .org/10.1001/jama. 195 1.03670270014006 